
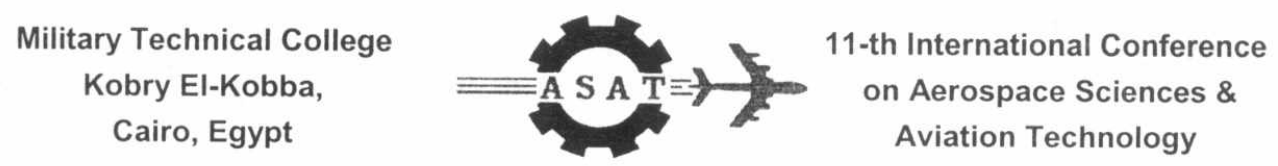

\title{
COMPUTATIONAL SIMULATION AND EXPERIMENTAL VALIDATION OF DOUBLE SQUARE CYLINDERS FLOW IN STAGGERED ARRANGEMENT
}

\author{
A. M. Abdel Dayem' and N. N. Bayomi ${ }^{2}$
}

\begin{abstract}
Experimental and numerical study of the flow field around two finite square cylinders in staggered arrangement is the objective of the present work. Two similar cylinders having the same square cross-section of $50 \mathrm{~mm} \times 50 \mathrm{~mm}$ with blockage ratio of $16.7 \%$ were considered in different arrangements. The staggered arrangement with different angular positions was considered. The turbulent incompressible NavierStokes equations are solved numerically using finite element method. The static pressure distributions on the two-cylinder surfaces were estimated and measured in all cases with different apart-distances between the two cylinders and consequently the pressure coefficient variations were calculated. In addition, the experiments were developed for three Reynolds numbers of 13.5, 9.5 and $6.8 \times 10^{4}$ (based on the cylinder side length) to investigate their effect on the turbulent flow. The upstream cylinder is not affected greatly for different angular positions and apart-distances. This effect is significant in the case of side-by-side, where the flow stream equally affects the two cylinders. Up to four cylinder-widths apart-distance, the upstream cylinder affects the downstream cylinder. The effect is changed from maximum value in tandem arrangement to minimum one for the case of side-by-side configuration. Moreover, where the upstream side of the downstream cylinder is affected by changing the Reynolds number, the other sides do not vary. The highest considered Reynolds number produces the lowest drag and side force coefficient. On the other hand, the computational results are in close agreement with the measured ones. The numerical visualization presents clearly the flow behavior around the cylinders and was validated by the measured and published data.
\end{abstract}

KEYWORDS: Double square cylinders, staggered arrangement, 2-D flow, Bluff bodies, CFD.

\section{INTRODUCTION}

The study of flow around two square cylinders placed in a main stream is of fundamental importance in fluid dynamics. Practical applications of this configuration are seen in various areas of engineering such as, heat exchangers, buildings, twin chimney stacks, electronic rods, parallel structures, porous medium, packages and off-shore structures mentioned among others.

\footnotetext{
'Mattaria, Helwan University, 11718 Masaken El-Helmia, Cairo, Egypt, adel_abdeldayem@hotmail.com

${ }^{2}$ Mattaria, Helwan University, 11718 Masaken El-Helmia, Cairo, Egypt, nnbayomi@hotmail.com
} 
One of the more intriguing topics in bluff body aerodynamics is the interaction of two bluff bodies placed in a staggered arrangement. The intriguing facet of this topic is that, the flow pattern and drag of a staggered configuration cannot be easily predicted from the known flow characteristics of the two individual bodies. The reason for this is that the rear body is exposed to a flow perturbed and substantially altered by the front body. When more than one bluff body is placed side by side in a uniform flow, the aerodynamic parameters and vortex-shedding parameters are completely different from the case of a single body, because their wakes or vortex streets interfere in a complex manner, depending on the spacing of the bars.

Wong, et al. [1] paper presented the results of the aerodynamic forces and vortex shedding of two square cylinders in side-by-side arrangement of size ratio $2: 1$ at sub-critical flow regime. Flow measurements were carried out at transverse spacing of $1.12 \leq T / L \leq 2.5$. T/L is the horizontal apart distance between the cylinders to the side length. The gap side shear layer of the large cylinder reattaches to its inner face for small separation, while the gap side shear layer of the small cylinder reattaches to its inner face for larger separation. The distributions of aerodynamic forces reveal that maximum pressure loading on the small cylinder occurs at a separation of $T / L=1.75$. No bistable flow is found within the transverse spacing studied.

Luo et al. [2] estimated experimentally the lift and drag acting on the downstream of two cylinders. The results are found to be in reasonable agreement (except in the range $S / L=3-4$ and $T / L=2-3$ whereas $S / L$ is the vertical apart distance between the cylinders to the side length) with previous measurements. Based on these steady flow results, the region where the downstream cylinder will become unstable to transverse galloping (static instability) were estimated and reported.

Valencia [3] developed a numerical simulation to explore the laminar fluid flow in a channel with two square bars mounted side by side to employ the approaching flow. Solving the continuity and the Navier-Stokes equations in two dimensions has captured the effects of vortex shedding. A numerical investigation was conducted by Rosales et al. [4] to analyze the unsteady flow field and heat transfer characteristics for a tandem pair of square cylinders in a laminar channel flow. The results showed that the drag coefficient and cylinder Nusselt number decrease as the heated cylinder approaches the wall.

The flow characteristics around two square cylinders in a tandem arrangement were studied experimentally by Liu and Chen [5]. Hysteresis with two discontinuous jumps was presented for all values of the Reynolds numbers studied when the spacing is varied in two different ways, one being a progressive increase and the other a progressive decrease. It was found that the hysteresis regime shifts rapidly towards smaller values of spacing as the Reynolds number is increased in the lower range, $\operatorname{Re}=2.0 \times 10^{3}-5.3 \times 10^{3}$, and then remains nearly unchanged between $\mathrm{Re}=$ $5.3 \times 10^{3}-1.6 \times 10^{4}$.

Lin et al. [6] investigated experimentally the characteristics of wake structures behind two square cylinders arranged side by side. The Reynolds number varied from 2500 to 8000 , and the net apart-distance ratio $(\delta / L)$ ( $\delta$ is center-to center distance between the cylinders) changes from 0 to 3.0. The distribution of velocity vector, the evolutions of vortex shedding frequency in the wakes of two square 
cylinders, the formation lengths behind two cylinders and the characteristics of the apart-distance flow are all addressed in detail.

In spite of these recent efforts, the effects of the transverse apart-distance of mounted bars in a turbulent channel flow have not been reported in the literature for different angular positions. Therefore, this work was undertaken to provide information on the flow processes in channels with two square bars arranged in different configurations to the approaching flow. It was decided to investigate the flow about two similar square cylinders due to the limited amount of work that has been developed in this area. Therefore, this paper investigates results of the flow around two finite-square cylinders with equal cross-sectional area in a confined region. Measurements and numerical estimation of the surface pressure distribution around the two cylinders were obtained. The cylinders apart-distances of $2 \mathrm{~L}, 3 \mathrm{~L}$ and $4 \mathrm{~L}(\mathrm{~L}=$ cylinder width) were studied. The cylinders aligned in parallel to each other and perpendicular to the flow. The two confined cylinders were supported from their two edges to study the flow as a two dimensional flow. Each cylinder is not rotated about its axis.

\section{EXPERIMENTAL TEST RIG AND INSTRUMENTATION}

The experiments were performed in an open wind tunnel, located in the laboratory of Fluid Mechanics, Faculty of Engineering at Mattaria, Helwan University. The wind tunnel consists of an air blower that is connected with a $600 \mathrm{~mm} \times 600 \mathrm{~mm}$ cross section and $10 \mathrm{~m}$ long with an opening as shown in Fig. 1. An extension of the tunnel with $2 \mathrm{~m}$ long was developed. The experimental mechanism was established inside the extension section to avoid the changing in the tunnel. The tunnel has a free stream turbulence intensity of about $2 \%$ as described by Sedrak and $\mathrm{EI}$ Dandoush [7] and a maximum speed of $9.5 \mathrm{~m} / \mathrm{s}$.

Two hollow aluminum cylinders were mounted between two wooden rotating discs of diameter $300 \mathrm{~mm}$ that were flushed with the floor and roof of the tunnel. Since the center of rotation of the turn discs was made to coincide with the center of the downstream cylinder, see Fig. 1, the rotation of the turn discs could produce a rather wide range of staggered arrangement when the distance between the cylinders changed periodically. Whereas the distance between the centerline of the upstream and downstream cylinders (denoted by $\delta$ ) was varied in the range from 2.0 $L$ to $4.0 \mathrm{~L}$, where $\mathrm{L}$ being the cylinder side length.

The two hollow cylinders that have equal square cross-section areas were used. The cylinders having smooth surfaces to avoid any flow obstacle that can change the flow direction. The cylinders were supported vertically from their edges along the roof and floor of the tunnel. The cylinders were perpendicular to the flow direction in all cases of rotation or Reynolds number with zero flow incident angle. In addition, the cylinders were fully tightened with the discs (tunnel walls) to prevent any dispersion of flow below or above the cylinders edges. Plate 1, presents the mechanism of the cylinders fixation with the wooden discs.

Two screw-bolts were used to fix the two cylinders between the two discs. This mechanism allows changing the angular position of the upstream cylinder by rotating the two discs into the specified angles. The two discs center was located in $500 \mathrm{~mm}$ 
apart from the tunnel opening. In addition, establishing different holes on the two discs by the specified distances allowed changing the apart-distance between the cylinders where the bolts fixed the cylinders through the holes. These holes were closed after preparing the case experiments. That means, for a certain apart-distance the data were measured for different angular positions by rotating the two discs before moving the upstream cylinder into a new location.

The wind flow speed was controlled through a gate located in the wind tunnel inlet. Changing the wind tunnel inlet opening can vary the flow velocity and accordingly the Reynolds number (Re) into three values of $13.5,9.5$ and $6.8 \times 10^{4}$. These values depend on the two cylinders dimensions of $50 \mathrm{~mm} \times 50 \mathrm{~mm}$ crosssection which are similar. These dimensions make a blockage ratio of $16.7 \%$ with the wind cross sectional area and a cylinder aspect ratio of 12. Pressure tapings were fixed on the surfaces of downstream and upstream cylinders at $100 \mathrm{~mm}$ apart from the cylinders upper edges (tunnel roof) to avoid the boundary layer effect. Seventytwo pressure-taps were distributed on the cylinders surfaces; nine taps on each side on a straight horizontal row. This distribution is clearly presented in Fig. 2, the taps are concentrated near cylinder corners to describe accurately the pressure in this significant area. The taps are located at the same horizontal plane on both cylinders sides to explain the flow at the same plane. The pressure was measured on both cylinders surfaces (upstream and downstream cylinders) to study the flow around both cylinders.

A Prandtl probe connected to a digital micromanometer with accuracy $\pm 1 \%$ was used to measure the air free stream velocity. The Prandtl probe detected the time averaged tunnel velocity distribution. The distance from uniformity of velocity distribution was so small. Moreover, the pressure tapings were connected to the digital micromanometer model Yokogawa 2655, with resolution of $0.1 \mathrm{~Pa}$ and updating of the reading every 0.4 second as shown in Fig. 1, to measure the local pressure at each point. The used digital micromanometer accuracy is 0.01 . An average of the readings is computed every 5 seconds using an A/D converter and a $\mathrm{PC}$ to obtain the average of fluctuated data.

The gauge pressure was measured in the specified location on the cylinder surfaces for one tap to another in clockwise direction using the digital micromanometer in the steady state flow with the same free stream conditions. Simultaneously the same procedure was repeated with other Reynolds number, displacement distance and angular position as explained in Fig. 2. Following measuring the tapping pressure, the pressure coefficient, drag force coefficient and side force coefficient were estimated for each cylinder simultaneously.

\section{COMPUTATIONAL MODELING}

A two-dimensional numerical modeling of the flow around the considered two cylinders was performed by the ANSYS 5.4 code that is based on the finite element technique. The computational domain presented latter in Figs. $9,10 \& 11$, is a rectangle of $0.9 \mathrm{~m}$ length and $0.60 \mathrm{~m}$ width (tunnel width). The upstream cylinder center is located at $x=0.3 \mathrm{~m}$ and $\mathrm{y}=0.3 \mathrm{~m}$ in the domain from the left.

First of all a grid generation of the domain was established with fine grids around the cylinder. The fine meshing around the cylinders can accelerate the solution 
convergence and avoid any solution divergence and errors in this critical area. Accordingly the grid distribution should be controlled inside the domain.

Three cases are considered for two apart distances $2 \mathrm{~L}$ and $4 \mathrm{~L}$. The cases are tandem, side-by-side and $45^{\circ}$ staggered configurations. The quadrilateral structured mesh was used for all cases considered. The numbers of grids developed as shown latter in Figs. 9, $10 \& 11$, are as in Table 1. However, the size of the elements is changed according to the concentration of the grids. Incompressible and turbulent flow was considered for all cases studied.

Table 1. Number of grids for all cases considered.

\begin{tabular}{|c|c|c|c|}
\hline $\begin{array}{c}\text { Apart } \\
\text { distance }\end{array}$ & Tandem & Side-by-side & Stagger $\left(45^{\circ}\right)$ \\
\hline 2L & 1226 & 3027 & 4912 \\
\hline $4 \mathrm{~L}$ & 2653 & 2009 & 2659 \\
\hline
\end{tabular}

For a Newtonian fluid, the relationship between the stress and rate of deformation of the fluid is:

where: $P=$ Flow pressure

$$
\tau_{\mathrm{ij}}=-\mathrm{P} \delta_{\mathrm{ij}}+\mu\left(\frac{\partial \mathrm{u}_{\mathrm{i}}}{\partial x_{\mathrm{j}}}+\frac{\partial \mathrm{u}_{\mathrm{j}}}{\partial x_{\mathrm{i}}}\right)
$$

$\mathrm{u}_{\mathrm{i}} \& \mathrm{u}_{\mathrm{j}}=$ Orthogonal velocities $\left(\mathrm{u}_{1}=\mathrm{V}_{\mathrm{x}}, \mathrm{u}_{2}=\mathrm{V}_{\mathrm{y}}, \mathrm{u}_{3}=\mathrm{V}_{\mathrm{z}}\right)$

$V_{x}, \& V_{y}=$ Components of the velocity vector in the $x, y$ directions, respectively $x \& y=$ Global Cartesian coordinates

$\delta_{\mathrm{ij}}=$ Kronecker Delta equals one if $\mathrm{i}=\mathrm{j}$ and equals zero for others.

$\mu=$ Dynamic viscosity

$\tau_{i j}=$ Stress tensor

\subsection{Continuity Equation}

From the law of conservation of mass law, it comes the continuity equation:

$$
\frac{\partial \rho}{\partial t}+\rho \frac{\partial\left(V_{x}\right)}{\partial x}+\rho \frac{\partial\left(V_{y}\right)}{\partial y}=0
$$

Where: $V_{x}$, and $V_{y}=$ Components of the velocity vector in the $x, y$ directions,

$$
\begin{aligned}
& \text { respectively } \\
\mathrm{x} \text { and } \mathrm{y} & =\text { Global Cartesian coordinates } \\
\mathrm{t} & =\text { Time } \\
\rho & =\text { Density }
\end{aligned}
$$

The rate of change of density can be replaced by the rate of change of pressure and the rate at which density changes with pressure:

$$
\frac{\partial \rho}{\partial t}=\frac{\partial \rho}{\partial p} \frac{\partial p}{\partial t}
$$


Where $p$ is the flow pressure. The evaluation of the derivative of the density comes from the equation of state. The pressure waves are assumed to be traveled infinitely fast throughout the domain $\left(\mathrm{d} \rho / \mathrm{dp}=10^{-5}\right)$

The momentum equations, without further assumptions regarding the properties, are as follows:

$$
\begin{array}{r}
\frac{\rho \partial \mathrm{V}_{\mathrm{x}}}{\partial \mathrm{t}}+\frac{\rho \partial\left(\mathrm{V}_{\mathrm{x}} \mathrm{V}_{\mathrm{x}}\right)}{\partial \mathrm{x}}+\frac{\rho \partial\left(\mathrm{V}_{\mathrm{y}} \mathrm{V}_{\mathrm{x}}\right)}{\partial \mathrm{y}}=\rho \mathrm{g}_{\mathrm{x}}-\frac{\partial \mathrm{P}}{\partial \mathrm{x}} \\
+\mathrm{R}_{\mathrm{x}}+\frac{\partial}{\partial \mathrm{x}}\left(\mu_{\mathrm{e}} \frac{\partial \mathrm{V}_{\mathrm{x}}}{\partial \mathrm{x}}\right)+\frac{\partial}{\partial \mathrm{y}}\left(\mu_{\mathrm{e}} \frac{\partial \mathrm{V}_{\mathrm{x}}}{\partial \mathrm{y}}\right)+\mathrm{T}_{\mathrm{x}} \\
\frac{\rho \partial \mathrm{V}_{\mathrm{y}}}{\partial \mathrm{t}}+\frac{\rho \partial\left(\mathrm{V}_{\mathrm{x}} \mathrm{V}_{\mathrm{y}}\right)}{\partial \mathrm{x}}+\frac{\rho \partial\left(\mathrm{V}_{\mathrm{y}} \mathrm{V}_{\mathrm{y}}\right)}{\partial \mathrm{y}}=\rho \mathrm{g}_{\mathrm{y}}-\frac{\partial \mathrm{P}}{\partial \mathrm{y}} \\
+\mathrm{R}_{\mathrm{y}}+\frac{\partial}{\partial \mathrm{x}}\left(\mu_{\mathrm{e}} \frac{\partial \mathrm{V}_{\mathrm{y}}}{\partial \mathrm{x}}\right)+\frac{\partial}{\partial \mathrm{y}}\left(\mu_{\mathrm{e}} \frac{\partial \mathrm{V}_{\mathrm{y}}}{\partial \mathrm{y}}\right)+\mathrm{T}_{\mathrm{y}}
\end{array}
$$

Where $g_{x} \& g_{y}=$ Components of acceleration due to gravity.

$R_{x} \& R_{y}=$ Distributed resistance.

$T_{x} \& T_{y},=$ Viscous loss terms.

$$
\mu_{\mathrm{e}}=\text { Effective viscosity. }
$$

$\rho=$ Flow density.

$$
\begin{aligned}
& \mathrm{T}_{\mathrm{x}}=\frac{\partial}{\partial x}\left(\mu \frac{\partial \mathrm{V}_{\mathrm{x}}}{\partial x}\right)+\frac{\partial}{\partial \mathrm{y}}\left(\mu \frac{\partial \mathrm{V}_{\mathrm{y}}}{\partial \mathrm{x}}\right) \\
& \mathrm{T}_{\mathrm{y}}=\frac{\partial}{\partial \mathrm{x}}\left(\mu \frac{\partial \mathrm{V}_{\mathrm{x}}}{\partial \mathrm{y}}\right)+\frac{\partial}{\partial \mathrm{y}}\left(\mu \frac{\partial \mathrm{V}_{\mathrm{y}}}{\partial \mathrm{y}}\right)
\end{aligned}
$$

Then the partial differential equations solved in the Launder and Spalding [8] twoequation model are as follows:

$$
\begin{aligned}
& \frac{\rho \partial \mathrm{k}}{\partial \mathrm{t}}+ \frac{\rho \partial\left(\mathrm{V}_{\mathrm{x}} \mathrm{k}\right)}{\partial \mathrm{x}}+\frac{\rho \partial\left(\mathrm{V}_{\mathrm{y}} \mathrm{k}\right)}{\partial \mathrm{y}}=\frac{\partial}{\partial \mathrm{x}}\left(\frac{\mu_{\mathrm{t}}}{\sigma_{\mathrm{k}}} \frac{\partial \mathrm{k}}{\partial \mathrm{x}}\right)+\frac{\partial}{\partial \mathrm{y}}\left(\frac{\mu_{\mathrm{t}}}{\sigma_{\mathrm{k}}} \frac{\partial \mathrm{k}}{\partial \mathrm{y}}\right) \\
&+\mu_{\mathrm{t}} \Phi-\rho \varepsilon+\frac{\mathrm{C}_{4} \beta \mu_{\mathrm{t}}}{\sigma_{\mathrm{t}}}\left(\mathrm{g}_{\mathrm{x}} \frac{\partial \mathrm{T}}{\partial \mathrm{x}}+\mathrm{g}_{\mathrm{y}} \frac{\partial \mathrm{T}}{\partial \mathrm{y}}\right) \\
& \frac{\rho \partial \varepsilon}{\partial \mathrm{t}}+\frac{\rho \partial\left(\mathrm{V}_{\mathrm{x}} \varepsilon\right)}{\partial \mathrm{x}}+\frac{\rho \partial\left(\mathrm{V}_{\mathrm{y}} \varepsilon\right)}{\partial \mathrm{y}}=\frac{\partial}{\partial \mathrm{x}}\left(\frac{\mu_{\mathrm{t}}}{\sigma \varepsilon} \frac{\partial \varepsilon}{\partial \mathrm{x}}\right)+\frac{\partial}{\partial \mathrm{y}}\left(\frac{\mu_{\mathrm{t}}}{\sigma_{\varepsilon}} \frac{\partial \varepsilon}{\partial \mathrm{y}}\right) \\
&+\mathrm{C}_{1} \mu_{\mathrm{t}} \frac{\varepsilon}{\mathrm{k}} \Phi-\mathrm{C}_{2} \rho \frac{\varepsilon^{2}}{\mathrm{k}}+\frac{\mathrm{C}_{1} \mathrm{C}_{\mu} \mathrm{C}_{3} \beta \rho \mathrm{k}}{\sigma_{\mathrm{t}}}\left(\mathrm{g}_{\mathrm{x}} \frac{\partial \mathrm{T}}{\partial \mathrm{x}}+\mathrm{g}_{\mathrm{y}} \frac{\partial \mathrm{T}}{\partial \mathrm{y}}\right)
\end{aligned}
$$

Where $k=$ Turbulent kinetic energy.

$\varepsilon=$ Turbulent kinetic energy dissipation rate.

$\Phi=$ Flow variable.

$\beta=$ Input quantity.

The two equation turbulence model of Launder and Spalding [8] is used to evaluate the turbulent viscosity $\left(\mu_{\mathrm{t}}\right)$ through the expression: 


$$
\mu_{\mathrm{t}}=\mathrm{C}_{\mu} \rho \frac{\mathrm{k}^{2}}{\varepsilon}
$$

The final term in each equation is used to model the effect of buoyancy. Launder and Spalding [8] provided default values for the various constants in the model, see Table 2.

Table 2. Values for various constants in the model

\begin{tabular}{|l|l|c|c|l|l|l|l|l|}
\hline Value & $\mathrm{C}_{1}$ & $\mathrm{C}_{2}$ & $\mathrm{C} \mu$ & $\sigma_{\mathrm{k}}$ & $\sigma \varepsilon$ & $\sigma_{\mathrm{t}}$ & $\mathrm{C}_{3}$ & $\mathrm{C}_{4}$ \\
\hline Default & 1.44 & 1.92 & 0.09 & 1 & 1.3 & 1 & 0 & 0 \\
\hline
\end{tabular}

\subsection{Numerical Solution}

The Diagonal Matrix Algorithm (TDMA) method is described in detail in Patankar [9]. Where any entries outside the tri-diagonal portion, the method of breaking the problem into a series of tri-diagonal problems are treated as source terms using the previous values. For a completely unstructured mesh, or an arbitrary numbered system, the method reduces to the Gauss-Seidel iterative method.

The inlet conditions have inlet velocities with a component in the $x$ direction of $9.5 \mathrm{~m} / \mathrm{s}$ where the velocity component in the $y$ direction equals to zero. The no-slip condition is applied at the upper and lower boundaries and on the surface of the cylinders. The outlet conditions of the domain were limited to atmospheric pressure (zero gauge pressure at the tunnel opening). These boundary conditions are enough to solve the governing equations to estimate the flow primitive variables; pressure and velocity for each element inside the computational domain. The solution of the governing equations was obtained iteratively starting with initial values of the variables to reach a converged solution. One hundred iterations were taken into consideration for all solved cases. This number of iterations assures a convergence and stable solution for all cases. The convergence solution took few minutes for all problems that reached up to 10 minutes.

\section{4- RESULTS AND DISCUSSION}

For the purpose of simplicity, the description of the experimental results will be made with reference to Fig. 2, in which the downstream cylinder, $A$ is tentatively assumed to be fixed on the disc center and the two parameters $\delta$ and $\theta$ (the angle between the cylinders center-to-center line and incident flow direction) suffice to determine the arrangement of the two cylinders. Tandem arrangement occurs when $\theta$ equals zero degree, while side-by-side arrangement corresponds to $\theta=90^{\circ}$. All other values of $\theta$ represent staggered arrangement. Moreover, it may be noted that cylinder A is the fixed downstream cylinder and cylinder B is the upstream cylinder for all considered angular positions.

Many experiments were performed to study any possible flow form around two square cylinders. Study of displacement distances and angular positions were taken in consideration. The arrangements include the staggered, tandem and side by side 
configurations. The apart-distance between the two cylinders is a function of the length of the cylinders side $(\mathrm{L})$. It was varied for $2 \mathrm{~L}, 3 \mathrm{~L}$ and $4 \mathrm{~L}$ until the effect of the staggered configuration was vanished. That can be understood when the pressure distribution is relatively the same for both two cylinders.

Different angular positions were also considered to study different possible flows with different angular angles $(\theta)$. The angles considered are $0^{\circ}, 15^{\circ}, 30^{\circ}, 45^{\circ}$ and $90^{\circ}$ as shown in Figs. 3 to 6 . Zero-flow-incident angles $(\varphi)$ was only considered for both two cylinders for all staggered arrangements. The pressure was measured and listed for each location simultaneously.

The strategy of the measuring set up was started by measuring of tapping pressure on the cylinders surfaces of considered angular positions and Reynolds numbers for $2 \mathrm{~L}$ apart, then for $3 \mathrm{~L}$ and finally for $4 \mathrm{~L}$. In brief, the measurements can present the effect of three parameters: (1) angular positions of the two cylinders, (2) the apart-distance between the two cylinders and (3) the flow Reynolds number. Those parameters cover any possible configuration of the cylinders flow. The presentation of such data is shown in the figures. Some cases of experiments were not established for the apart-distance of $4 \mathrm{~L}$ due to the insignificant effect of staggered configuration on the pressure measurement. This is clearly shown for $45^{\circ}, 60^{\circ}, 75^{\circ}$ and $90^{\circ}$ angular positions.

The pressure coefficient $(\mathrm{Cp})$ was estimated for each location. The pressure coefficient $(C p)$ is defined as the ratio of the pressure difference between the local pressure $(P)$ and total pressure $\left(P_{\infty}\right)$ to the dynamic pressure. To investigate the forces on the cylinders surfaces there are two types of forces. The drag force acting in the flow direction perpendicular to the surfaces resulting from the flow pressure. This force can be defined in dimensionless form through the drag coefficient $\left(C_{D}\right)$. The second force acting on the cylinders is the side force resulting from the unequal distribution of pressure on side walls. The dimensionless form of this force is called the side force coefficient $\left(\mathrm{C}_{\mathrm{S}}\right)$.

The pressure coefficient was estimated for all surfaces of both cylinders as shown in the Figs. 3, 4, 5, \& 6, for the two considered Reynolds numbers. These figures investigate the significance of the angular position, apart-distance between the two cylinders and different Reynolds numbers on the flow. Those parameters significantly affected the flow around the two cylinders. Since the figures indicate the effect of the distance and angle between the two cylinders, Fig. 7, explains the relation of $\mathrm{Cp}$ versus $\mathrm{Re}$ and the apart-distance between the two cylinders for tandem configuration.

Figure 3 , represents pressure coefficient distribution $(C p)$ estimated for the surfaces of the upstream cylinder B for the considered angular positions $(\theta)$ and apart-distances $(\delta)$. They were estimated for the first Reynolds number $\operatorname{Re}_{1}$. The pressure coefficient distribution $(\mathrm{Cp})$ of the $\overline{\mathrm{CD}}$ side is only affected by the flow between the cylinders in the case of tandem arrangement $\left(\theta=0^{\mathrm{O}}\right)$ while the other sides have a negligible change. For the other angular positions, the upstream side $\overline{\mathrm{AB}}$ (the far side from the downstream cylinder) is negligibly changed (less than $5 \%$ change from single cylinder flow). The other sides are relatively modified (in general up to $20 \%$ change from single cylinder flow). The pressure coefficient distribution 
(Cp) of the downstream side $\overline{\mathrm{CD}}$ seems to be reduced with increasing the apartdistance between the two cylinders. Generally, the stagger effect is not large for the case of $\mathrm{Re}_{1}=13.5 \times 10^{4}$. This effect is clearer for the side $\overline{\mathrm{DA}}$ (which is greatly modified for the angular positions of $45^{\circ}$ and $60^{\circ}$ for $\delta=2 \mathrm{~L}$ ), those is fully interacted with the downstream cylinder $\mathrm{A}$.

As shown in Fig. 4, the pressure coefficient distribution (Cp) of the downstream cylinder $\mathrm{A}$ was drawn for the Reynolds number $\mathrm{Re}_{1}$. Its distribution was obtained for different $\theta$ and $\delta$. The effect of the staggered arrangement is more clear (the $\mathrm{Cp}$ is about $30 \%$ changing from single cylinder flow) here than in the case of upstream cylinder compared with the single cylinder in Fig. 7. The pressure coefficient is changed widely with increasing the apart-distance between two cylinders especially in the cases of mid angular positions located between $45^{\circ}$ and $75^{\circ}$ angular positions, it has negative values for $0^{\circ}$ with $\delta=2 \mathrm{~L}$. Thereafter, the stagger effect is generally vanished after $3 \mathrm{~L}$ apart-distance. In addition, the upstream side $(\overline{12})$ of the downstream cylinder $A$ is obviously affected for the cases of $0^{\circ}$ and $15^{\circ}$ angular positions. In this case, this side is located in the wake region of the upstream cylinder B.

Similar results of the upstream cylinder B are obtained for the two cases of the Reynolds numbers $\mathrm{Re}_{2}$ and $\mathrm{Re}_{3}$. The pressure coefficient of the upstream cylinder for $\mathrm{Re}_{3}$ is shown in Fig. 5. The effect of the Reynolds number changing on the $\mathrm{Cp}$ is little (up to $20 \%$ change from single cylinder flow) and this is because the flow is in a fully developed turbulent mode. The distribution of pressure coefficient is similar to corresponding cases of different Reynolds numbers. Figure 6, indicates the pressure coefficient of the downstream cylinder $A$ for Reynolds numbers $\mathrm{Re}_{3}$. As discussed in Fig. 4, the pressure coefficient is changed with $\theta$ and $\delta$. In general, it has a similar profile as $\mathrm{Re}_{1}$ case. The difference in the pressure coefficient of the two $\mathrm{Re}$ cases may be insignificant. This proves that again the experiments were performed in a fully developed turbulent flow as reported by Liu and Chen [5].

Figure 7 , shows clearly the effect of the considered Reynolds numbers $\operatorname{Re}_{1}$, $\mathrm{Re}_{2}$, and $\mathrm{Re}_{3}$ on the pressure coefficient of tandem configuration for different apartdistances. These cases are compared in the figure with the case of single cylinder flow that is located at the top of the figure for the corresponding apart-distances. As indicated before, the effect of changing the Reynolds number is insignificant (it ranges in $10 \%$ ) for the range considered. The data are presented only for the tandem configuration and the same conclusion can be found for the other cases. The apartdistance $(\delta)$ only affects the pressure coefficient for different angular positions. It can be seen also in Fig. 7, that the influence of the apart-distance is relatively vanished after $4 \mathrm{~L}$-separation distance between the two cylinders.

Generally, the effect of the staggered arrangement is relatively vanished after the apart-distance of $3 \mathrm{~L}$ for all cases considered. This is correct for both upstream and downstream cylinders. This notice is clearly explained for $45^{\mathrm{O}}$ angular position where the biggest apart-distance is obtained. In addition the downstream cylinder A is largely affected in tandem arrangement while the effect of Reynolds number is not large. The manner in which the pressure distribution changes as $\theta$ increased from zero degree to the angle at which the common distribution is established depending on the apart-distance. 
As shown in Figs. 4 \& 6 , the upstream side $(\overline{12})$ of the downstream cylinder is affected largely for tandem configuration, $\theta=0^{\circ}$, it changes up to $50 \%$ for $\delta=2 \mathrm{~L}$ and this effect is decreased gradually with angular positions. This effect is relatively vanished for the side-by-side configuration. In addition, the staggered effect is gradually decreased with increasing the effect of Re, the flow is uniform changed from $\mathrm{Re}_{1}$ to $\mathrm{Re}_{2}$. The flow change has relatively the same variation from position to another.

The downstream cylinder $A$ in tandem configuration is highly affected by the upstream cylinder $B$ until $\delta=4 \mathrm{~L}$. This influence is decreased when the angle $\theta$ changing. The smallest effect can be obtained for the configuration of side-by-side where the two cylinders have the same flow profile. This conclusion is clearly shown at the apart-distance $(\delta)$ of $3 \mathrm{~L}$ where the staggered influence is vanished. Since this effect is relatively vanished after $\delta$ equals $4 \mathrm{~L}$ (four cylinder widths) for the staggered arrangement. The same result can be obtained for the three Reynolds numbers considered in the study.

The pressure coefficient is greatly changed on the sides of $\overline{12}$ and $\overline{23}$ for the downstream cylinder A. These sides are in full contact with the upstream cylinder. They are influenced by the flow around the upstream cylinder. The other two sides $(\overline{34}$ and $\overline{41}$ ) are largely affected only in the case of tandem where the downstream cylinder is fully located in the wake region of the upstream cylinder. For other arrangements, the two sides are slightly affected by the stagger flow.

The Cp is well validated with the reported data of Liu and Chen [5] for both upstream and downstream cylinders as presented in Fig. $5 \& 6$. They obtained Cp for $2 \mathrm{~L}$ apart distance at flow of $1.6 \times 10^{4}$ Reynolds number to the cylinders in tandem configuration.

The parameters considered include Reynolds number (Re), angular positions and apart-distance $(\delta)$. The results indicate how far the location pressure from a corresponding pressure for a single cylinder flow. They explain clearly that the stagger effect decreased for wide apart-distance. Accordingly it is decreased with the apart-distance $(\delta)$ for all Reynolds numbers. It can be seen also that the influence of stagger is bigger for high angular angles than for small ones. The stagger effect is large for tandem arrangement and decreased gradually with the angular positions till it reaches $45^{\circ}$. Later on the influence increased again with increasing angular position $(\theta)$ till it equals $90^{\circ}$.

The drag force coefficient $\left(C_{D}\right)$ and side force coefficient $\left(C_{S}\right)$ were estimated. They were presented versus the angular position $(\theta)$ as shown in Fig. 8 . These coefficients were obtained for the three considered Reynolds numbers 13.5, 9.5, and $6.8 \times 10^{4}$. As expected the lower Reynolds number has higher $C_{D}$ where it has lower $\mathrm{C}_{\mathrm{s}}$. This is due to the higher static effect of the lower Reynolds number. For small angles of stagger and for small apart-distance, there are noticeable changes in the drag force as $\theta$ increases from zero degree as the base pressure increase to the angle of 90 where the side-by-side case. At the larger angles of stagger the behavior of the drag force simply reflects the behavior of the base pressure. The results are relatively accepted compared with the data of Lue \& Teng [10] and Luo et al. [2] as shown in Fig. 8. 
In summary, on decreasing the blockage for different Reynolds numbers $C_{D}, C_{S}$ and $\mathrm{Cp}$ decrease, while the stagnation pressure increases. These changes are all similar to a decrease in the Reynolds number corresponding to the fact that an increase in blockage is effectively an increase in the oncoming velocity.

\section{VERIFICATION OF THE NUMERICAL SIMULATION}

The experimental and numerical setup were carried out as discussed above. The numerical cases were validated by experimental ones as shown in Figs. $3 \& 4$. The cases considered in the numerical simulation are the tandem, side by side and $45^{\circ}$ angular angle, The cases were developed for $2 \mathrm{~L}$ and $4 \mathrm{~L}$ apart distances between the two cylinders. The first Reynolds number was only considered in the numerical work because the effectiveness of its changing on the flow can be ignored. In addition, after the apart-distance of $4 \mathrm{~L}$ the stagger effect is vanished. The pressure contours and velocity vectors along the considered domain are the numerical results presented in the Figs. 9 to 11. The mesh generation is presented also in the figures for each configuration. It changes depending on the apart distances between the cylinders. The grids are refined around the two cylinders and between them.

The pressure coefficient was estimated numerically on the cylinders surfaces and compared with the measured data. The numerical data are in excellent agreement with the corresponding measured ones as presented in Fig. $3 \& 4$. The small difference obtained between the measured and computed data was produced from the errors in the measurements and the boundary conditions. In addition, using of Launder and Spalding turbulence-model may obtain certain of accuracy near the walls. Moreover, the errors result from the flow fluctuations inside the wind tunnel and the accuracy limitation of the measuring tools. Accordingly, the boundary conditions could not specified correctly in the computational model. They were considered constant on the domain boundaries and this assumption is not practically true. In general, the difference between the experimental and numerical data can be accepted and it is in the range of $5 \%$.

The numerical flow visualization or velocity vector was developed as shown in the Figs. 9c, 9f, 10c, 10f, 11c \& 11f. They presented carefully the flow pattern between the two cylinders and past them. The apart-distance has a significant effect on the flow behaviour as can be seen in the figures described. They present also the weak effect the cylinders have on each other at $4 \mathrm{~L}$ apart-distance. After this distance the stagger effect will vanish. The wake region past the two cylinders is clear and it extends into about $2 \mathrm{~L}$ distance behind each cylinder. It is obvious that there is no eddies in the wake area, this is due to low flow velocity that considered.

The pressure contours were drawn inside the computational domain in the Figs. $9 \mathrm{~b}, 9 \mathrm{e}, 10 \mathrm{~b}, 10 \mathrm{e}, 11 \mathrm{~b} \& 11 \mathrm{e}$. They show obviously the stagnation pressures on the upstream face of the two cylinders and the pressure is lowered on the other sides, the downstream area has the lower pressure values as expected. In the far region of the two cylinders, the flow pressure is not affected and it equals the free stream pressure. In the cases of $4 \mathrm{~L}$ apart-distance the pressure in the area in between is lowered than in the cases of $2 \mathrm{~L}$ apart distance. Moreover, the pressure values on the cylinders sides are high where they decrease in far from the cylinder sides. 
As shown in Figs. 9 a, b \& c, the two cylinders in the tandem arrangement with apart-distance $(\delta)$ that equals $2 \mathrm{~L}$. The downstream cylinder $A$ is located in the wake flow region of the downstream cylinder, so its pressure distribution is largely affected especially in the upstream side of it. The upstream side has higher negative values of pressure. This behavior is relatively changed in the case of $45^{\circ}$ arrangement as clearly presented in Figs. 11 a, b \& c, while the flow is completely changed in Figs. $10 \mathrm{a}, \mathrm{b} \& \mathrm{c}$, where the side-by side case is presented. The flow is similar for each cylinder in this case and the pressure contours has similar values at the corresponding positions.

The other angular positions of the two cylinders that were not considered in the numerical simulation can be considered as a step of flow changing between the tandem and $45^{\circ}$ staggered or between the $45^{\circ}$ staggered and side-by-side configurations. The pressure values will increase on the downstream cylinder $A$ until they reach the maximum values at side-by side position where it is the lowest effect of stagger.

\section{CONCLUSION}

Experimental and numerical study of the flow around two finite similar square cylinders was established for different configurations of angular positions $(\theta)$ and apart-distances $(\delta)$ at three Reynolds numbers in the turbulent flow region. The pressure was obtained on the surfaces of downstream and upstream cylinders for different locations on the surfaces. Confined two cylinders were considered to study the flow in two-dimensional analysis. Tandem, staggered arrangements and side-byside configurations were taken in consideration for $2 \mathrm{~L}, 3 \mathrm{~L}$ and $4 \mathrm{~L}$ ( $\mathrm{L}=$ cylinder side length) apart-distances. The angular position, angle $\theta$, was changed also for $15^{\circ}, 30$ $\mathrm{O}, 45^{\circ}, 60^{\circ}$ and $75^{\circ}$ to study all expected flow cases. The pressure coefficient, force drag coefficient and side force coefficient were also estimated for the cases considered. As presented in the results, a significant effect of the upstream cylinder on the downstream cylinder was obtained in the tandem configuration. This effect is decreased with the angle $\theta$ till it reaches $90^{\circ}$; the side-by-side arrangement. This influence is vanished after $3 \mathrm{~L}$ apart-distance for all cases unless in the tandem case where the effect of staggered is expected to be neglected after $4 \mathrm{~L}$ distance. The measured ones successfully validated the computational results. The computational visualization is in close agreement with the experiments and previous published results. 


\begin{tabular}{|c|c|c|c|}
\hline \multicolumn{4}{|c|}{ NOMENCLATURE } \\
\hline A & Cylinder surface area & $x$ & $\begin{array}{l}\text { Distance from the side } \\
\text { edge }\end{array}$ \\
\hline$C_{D}$ & Drag force coefficient & $x \& y$ & $\begin{array}{l}\text { Global Cartesian } \\
\text { coordinates }\end{array}$ \\
\hline $\mathrm{C}_{\mathrm{p}}$ & Pressure coefficient & & \\
\hline $\mathrm{C}_{\mathrm{s}}$ & Side force coefficient & Symbol & \\
\hline $\mathrm{g}_{\mathrm{x}} \& \mathrm{~g}_{\mathrm{y}}$ & $\begin{array}{l}\text { Components of } \\
\text { acceleration due to gravity }\end{array}$ & $\delta$ & $\begin{array}{l}\text { Apart-distance between the } \\
\text { cylinders (center-to center) }\end{array}$ \\
\hline $\mathrm{K}$ & Turbulent kinetic energy & $\varepsilon$ & Turbulent kinetic energy \\
\hline L & Cylinder side length & & dissipation rate \\
\hline $\mathrm{P}$ & Local tapping pressure & $\Phi$ & Flow variable \\
\hline$P_{\infty}$ & Free stream pressure & $\mu$ & Dynamic viscosity \\
\hline $\mathrm{R}_{\mathrm{x}} \& \mathrm{R}_{\mathrm{y}}$ & Distributed resistance & $\mu_{\mathrm{e}}$ & Effective viscosity \\
\hline $\begin{array}{l}\mathrm{Re} \\
\mathrm{S} \& \mathrm{~T}\end{array}$ & $\begin{array}{l}\text { Reynolds number } \\
\text { Vertical and horizontal } \\
\text { distances between centers } \\
\text { of the two cylinders }\end{array}$ & $\begin{array}{l}\mu_{\mathrm{t}} \\
\theta \\
\varphi \\
\rho\end{array}$ & $\begin{array}{l}\text { Turbulent viscosity } \\
\text { Angular position angle } \\
\text { Incidence angle } \\
\text { Flow density }\end{array}$ \\
\hline $\begin{array}{l}\mathrm{T}_{\mathrm{x}} \& \mathrm{~T}_{\mathrm{y}} \\
\mathrm{U}_{\infty}\end{array}$ & $\begin{array}{l}\text { Viscous loss terms } \\
\text { Free stream velocity }\end{array}$ & $\tau_{i j}$ & Stress tensor \\
\hline$u_{1}$ & Orthogonal velocities & & \\
\hline$V_{x}, \& V_{y}$ & $\begin{array}{l}\text { Components of the velocity } \\
\text { vector in the } \mathrm{x}, \mathrm{y} \\
\text { directions, respectively }\end{array}$ & & \\
\hline
\end{tabular}

\section{REFERENCE}

[1] Wong, P. T. Y., Ko, N. W. M., and Chiu, A.Y.W., "Flow characteristics around two parallel adjacent square cylinders of different sizes", Journal of Wind Engineering and Industrial Aerodynamics, Vol. 54, pp. 263-275, (1995).

[2] Luo, S.C., Li, L. L. and Shah, D. A., "Aerodynamic stability of the downstream of two tandem square-section cylinders", J. of Wind Engineering and Industrial Aerodynamics, Vol. 79, pp. 79-103, (1999).

[3] Valencia A., "Laminar flow past square bars arranged side by side in a plane channel," cabierta.uchile.cl/revista/16/articulos/pdf/paper2, (2001).

[4] Rosales, J. L., Ortega, A. and Humphrey, J. A. C., "A numerical simulation of the convective heat transfer in confined channel flow past square cylinders: comparison of inline and offset tandem pairs," I. J. of Heat and Mass Transfer, Vol. 44, Issue 3, February, pp. 587-603, (2001).

[5] Liu, C. H. and Chen, J. M., "Observations of hysteresis in flow around two square cylinders in a tandem arrangement," J. of Wind Engineering and Industrial Aerodynamics, Vol. 90, Issue 9, September, pp. 1019-1050, (2002).

[6] Lin, C., Lin, W., Pai, C. and Shih, C., "Experimental study on wake structures behind two square cylinders arranged side by side using PIV and FLDV," Journal of Engineering, National Chung Hsing University, Vol. 14, No. 1, pp. 1-19, (2003).

[7] Sedrak, M. and El-Dandoush, S., "Effect of slenderness ratio and wind direction on bluff body aerodynamics,' Eng. Res. Bulletin, Helwan University, Vol. 7, pp. 54-77, (1989).

[8] Launder, B. E. and Spalding, D. B., "The numerical computation of turbulent flows," Computer Methods in Applied Mechanics and Engineering, Vol. 3, pp. 269289, (1974). 
[9] Patankar, S. V., "Numerical heat transfer and fluid flow," Hemisphere, New York, (1980)

[10] Lue, S. C. and Teng, T. C., "Aerodynamic forces on a square section cylinder that is downstream to an identical cylinder," Aerodynamic J. 6, pp. 203-212, (1990).

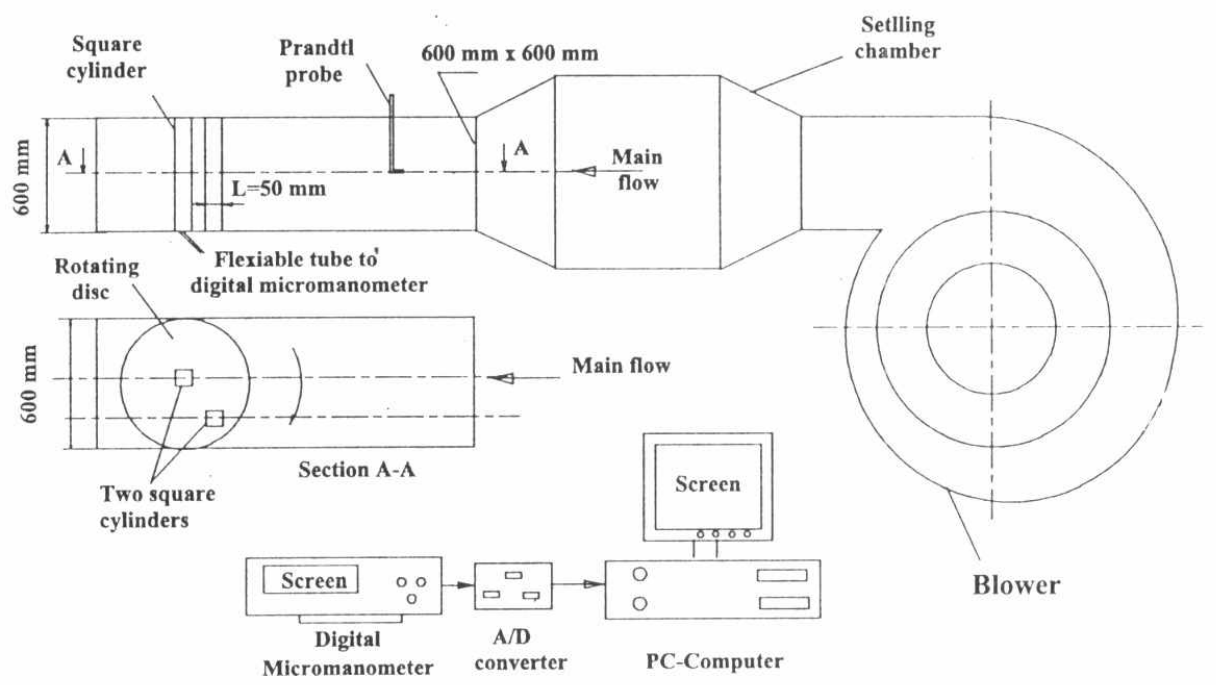

Fig. 1. Schematic diagram of the test rig

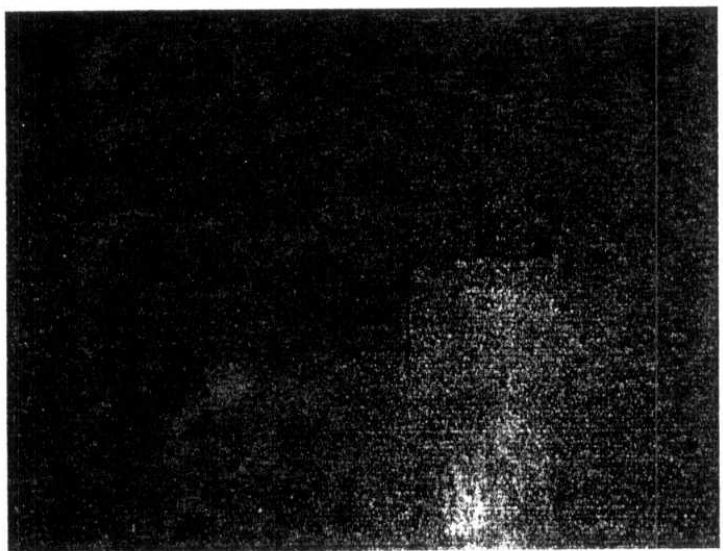

Plate 1. Photograph of the two cylinders rotating mechanism. 


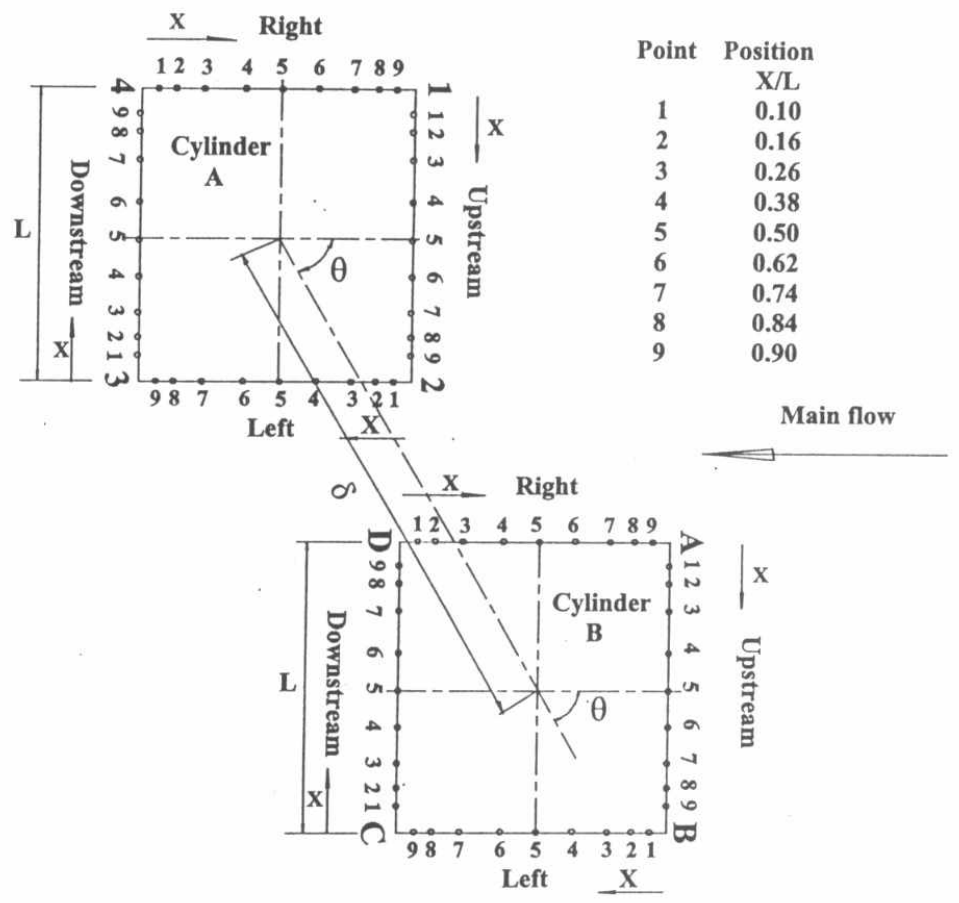

Fig. 2. Location of the static taps and configuration of the two cylinders. 

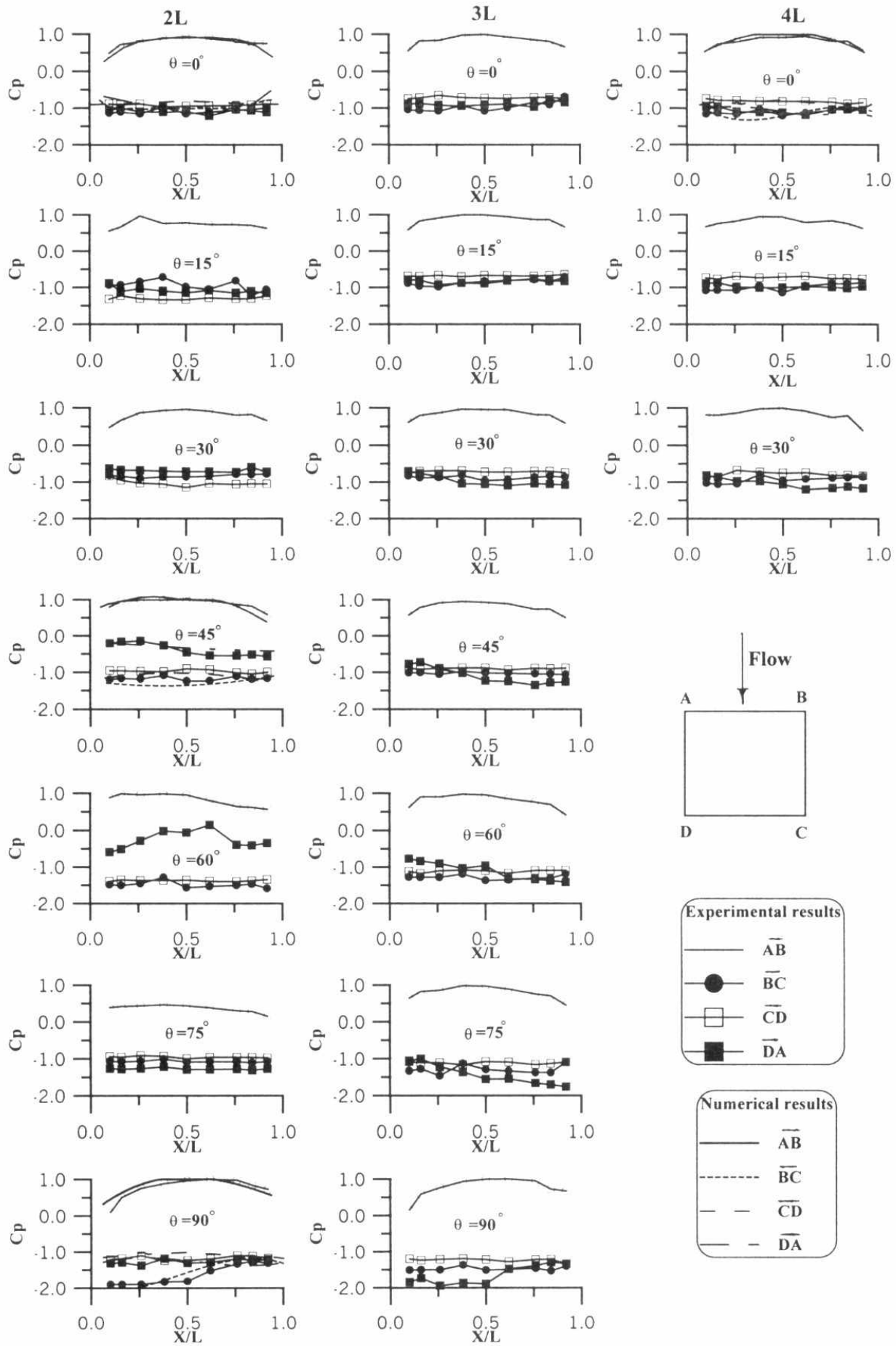

Fig. 3. Effect of angular position of two cylinders with various gaps at $\operatorname{Re}_{1}=13.5 \times 10^{4}$ for the upstream cylinder $\mathrm{B}$. 

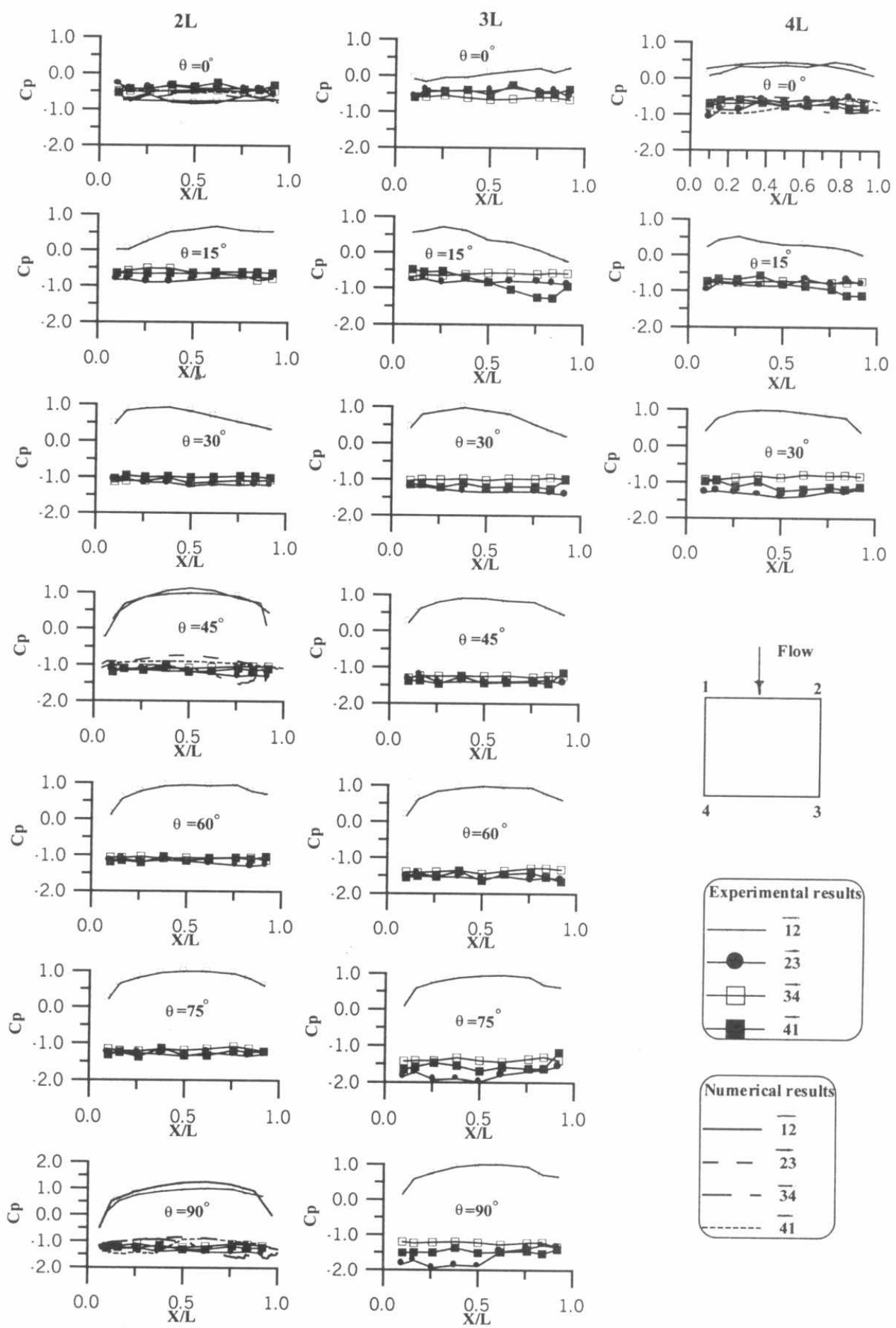

Fig. 4. Effect of angular position of two cylinders with various gaps at $\operatorname{Re}_{1}=13.5 \times 10^{4}$ for the downstream cylinder $A$. 

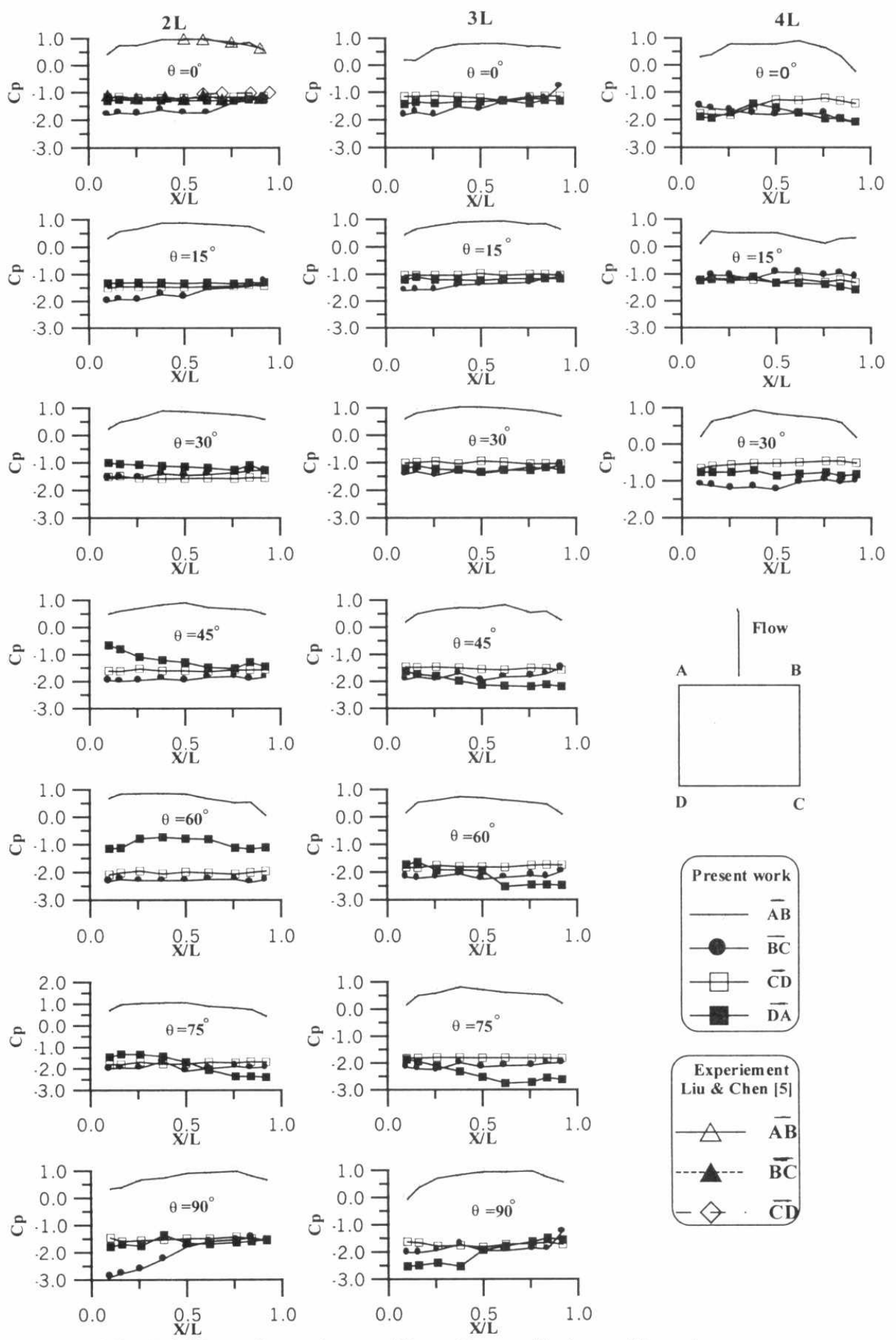

Fig. 5. Effect of angular position of two cylinders with various gaps at $\mathrm{Re}_{3}=6.8 \times 10^{4}$ for the upstream cylinder $\mathrm{B}$. 
$2 \mathrm{~L}$
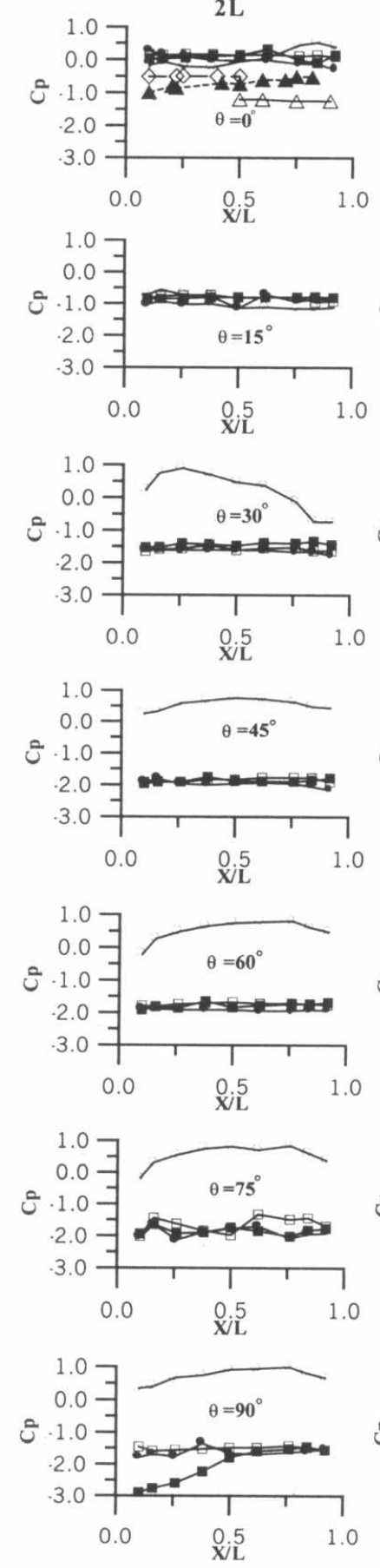

$3 \mathrm{~L}$
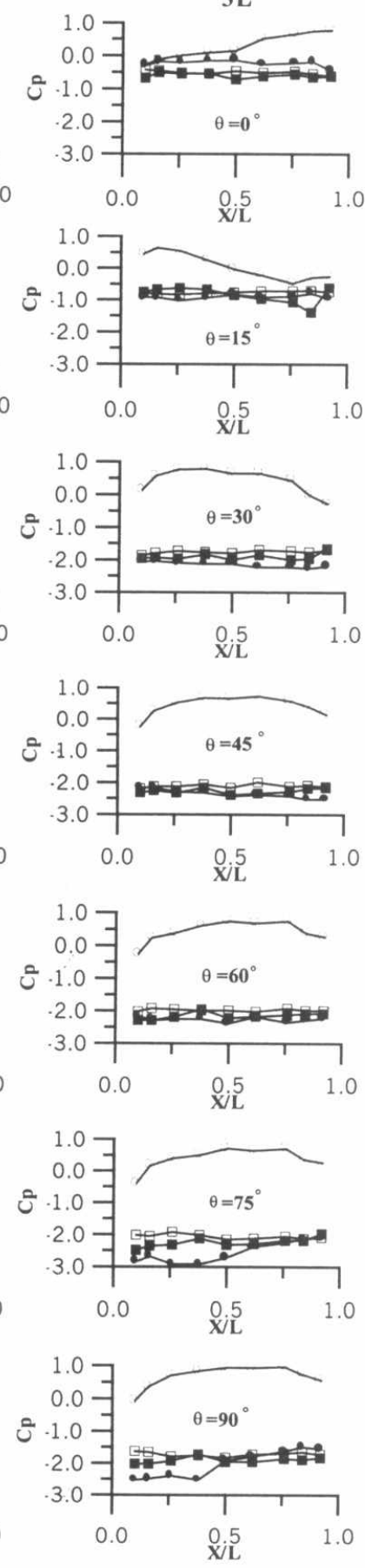

$4 \mathrm{~L}$
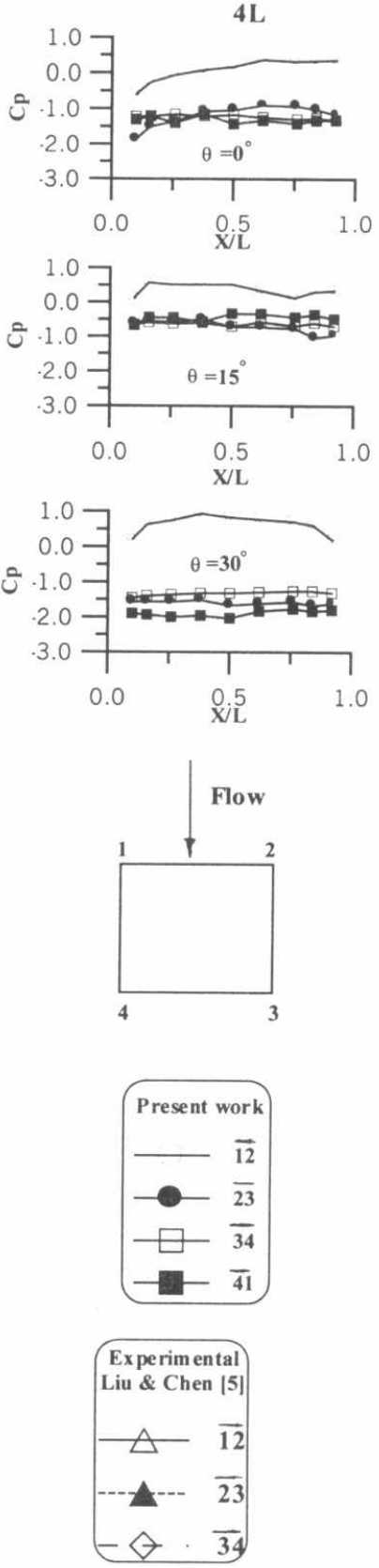

Fig. 6. Effect of angular position of two cylinders with various gaps at $\operatorname{Re}_{3}=6.8 \times 10^{4}$ for the downstream cylinder $\mathrm{A}$. 

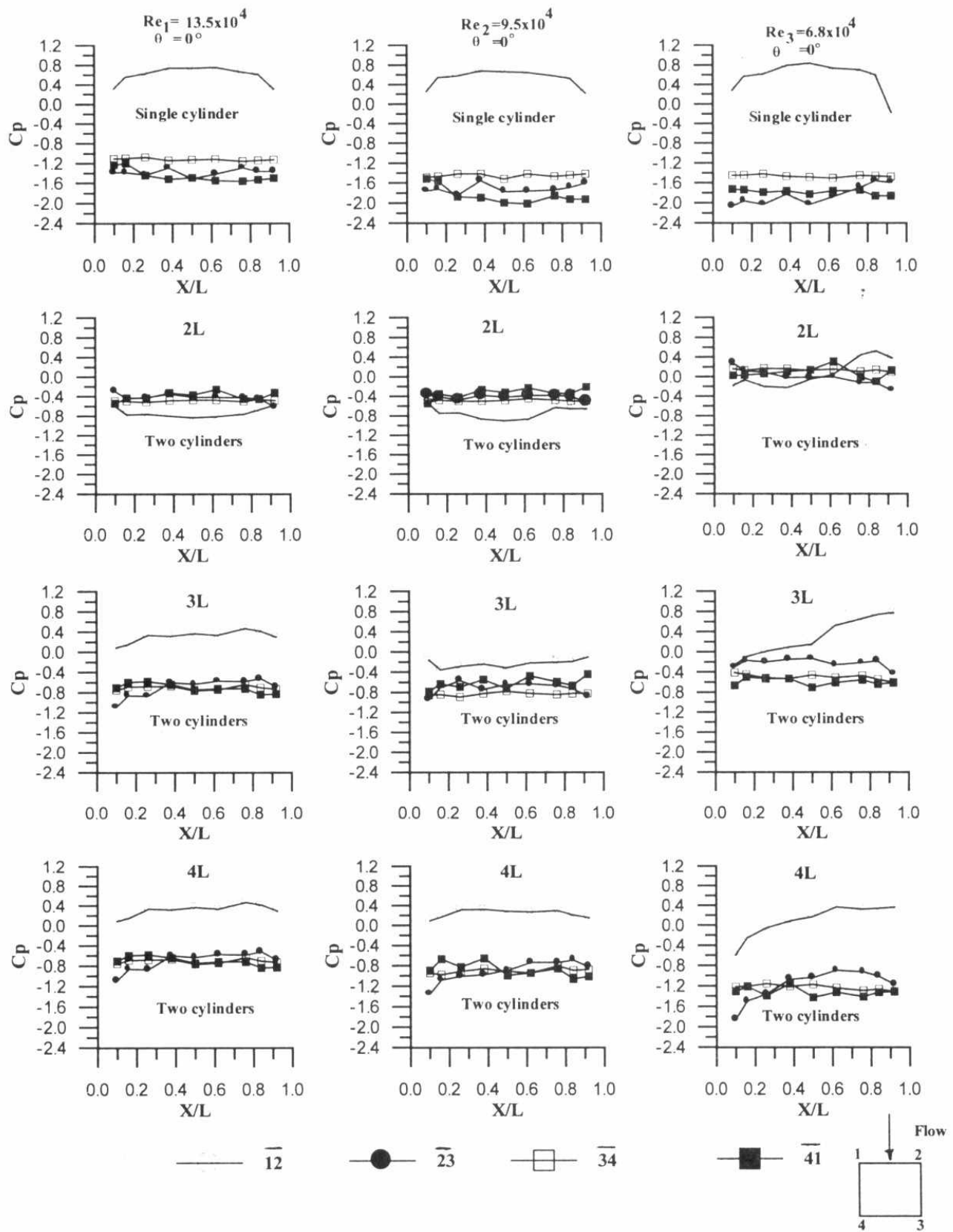

Fig. 7. Effect of distance between cylinders and Reynolds number on the pressure coefficient of the downstream cylinder $A$ for tandem configuration. 


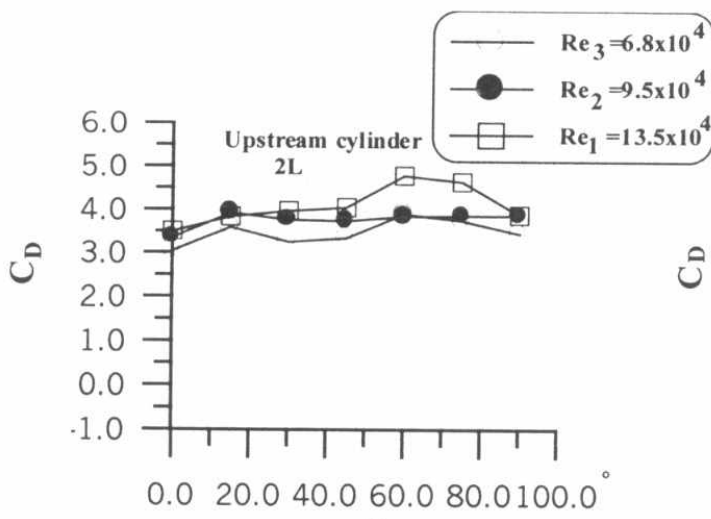

$\theta$

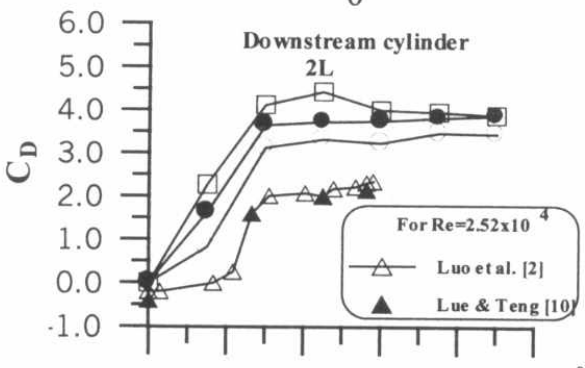

0.020 .040 .060 .080 .0100 .0
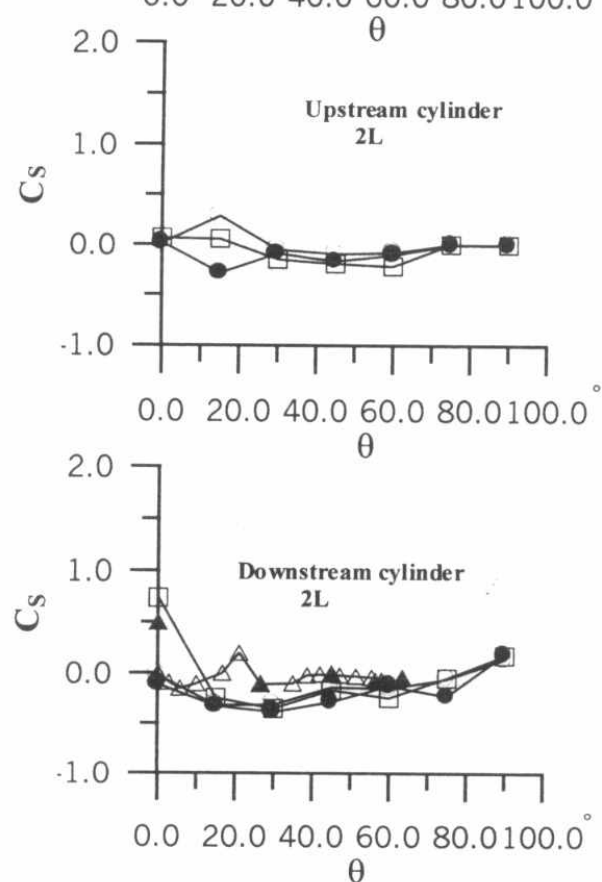

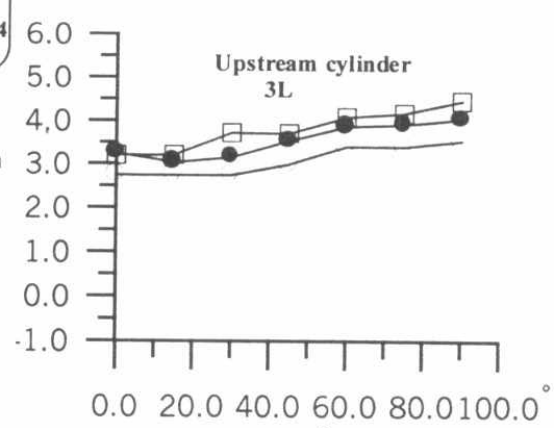

$\theta$

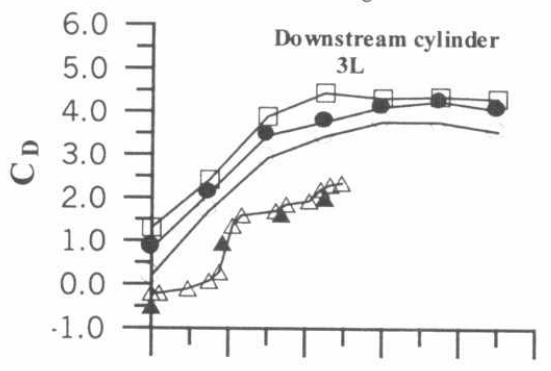

$0.020 .040 .060 .080 .0100 .0^{\circ}$
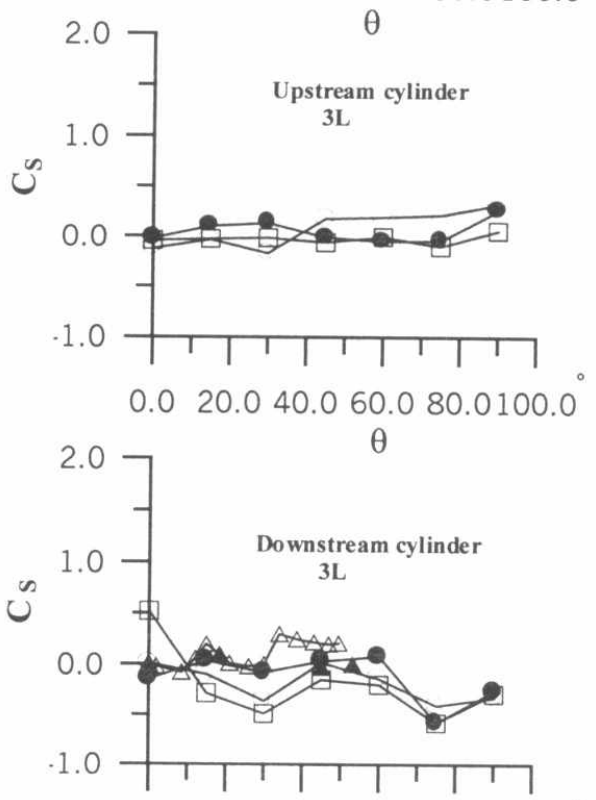

$0.020 .040 .060 .080 .0100 .0^{\circ}$

$\theta$

Fig. 8. Effect of angular position of two cylinders on the drag and side force coefficient with various Reynolds numbers. 


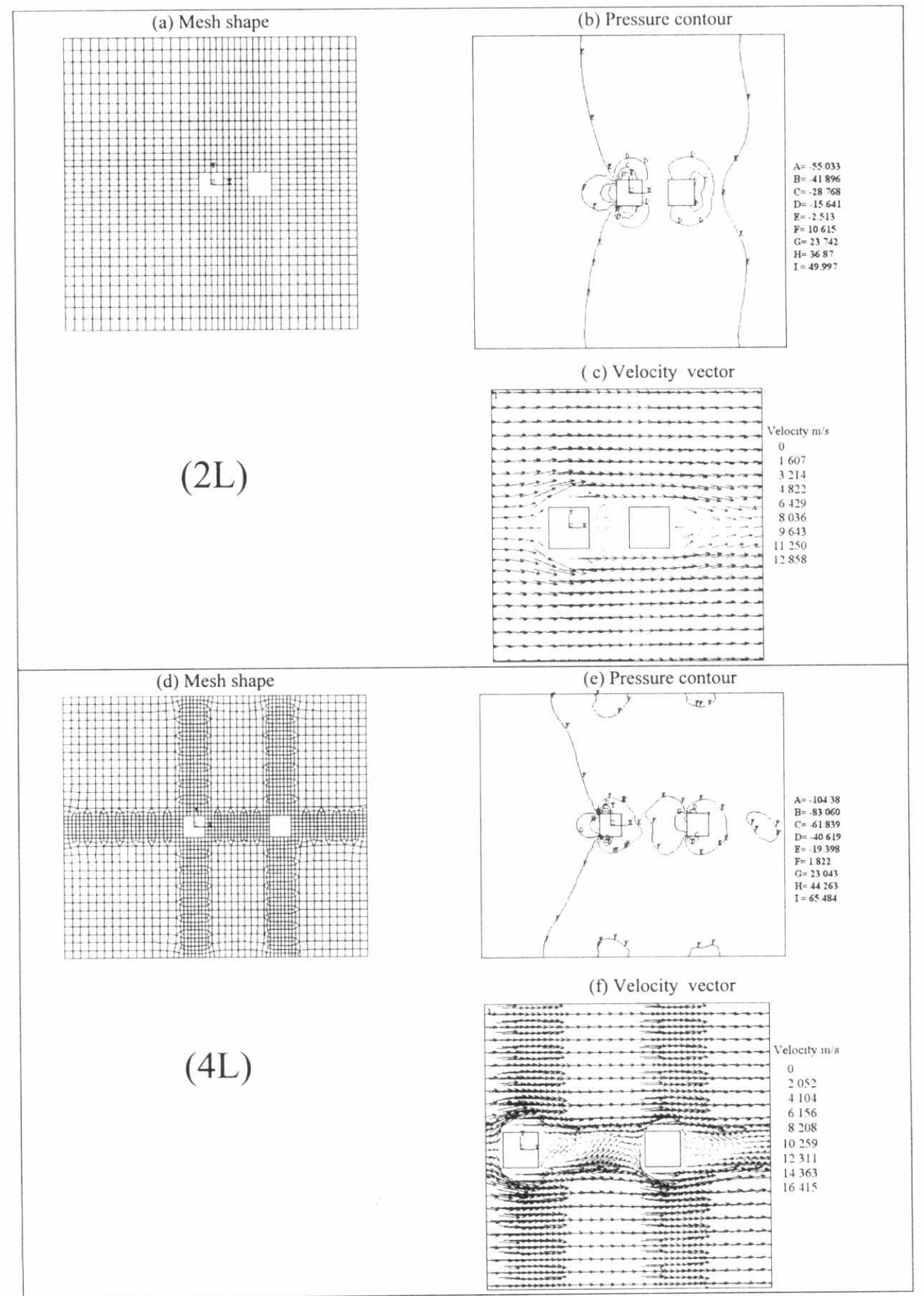

Fig. 9. Numerical results of tandem configuration for apart distances $2 \mathrm{~L}$ and $4 \mathrm{~L}$. 


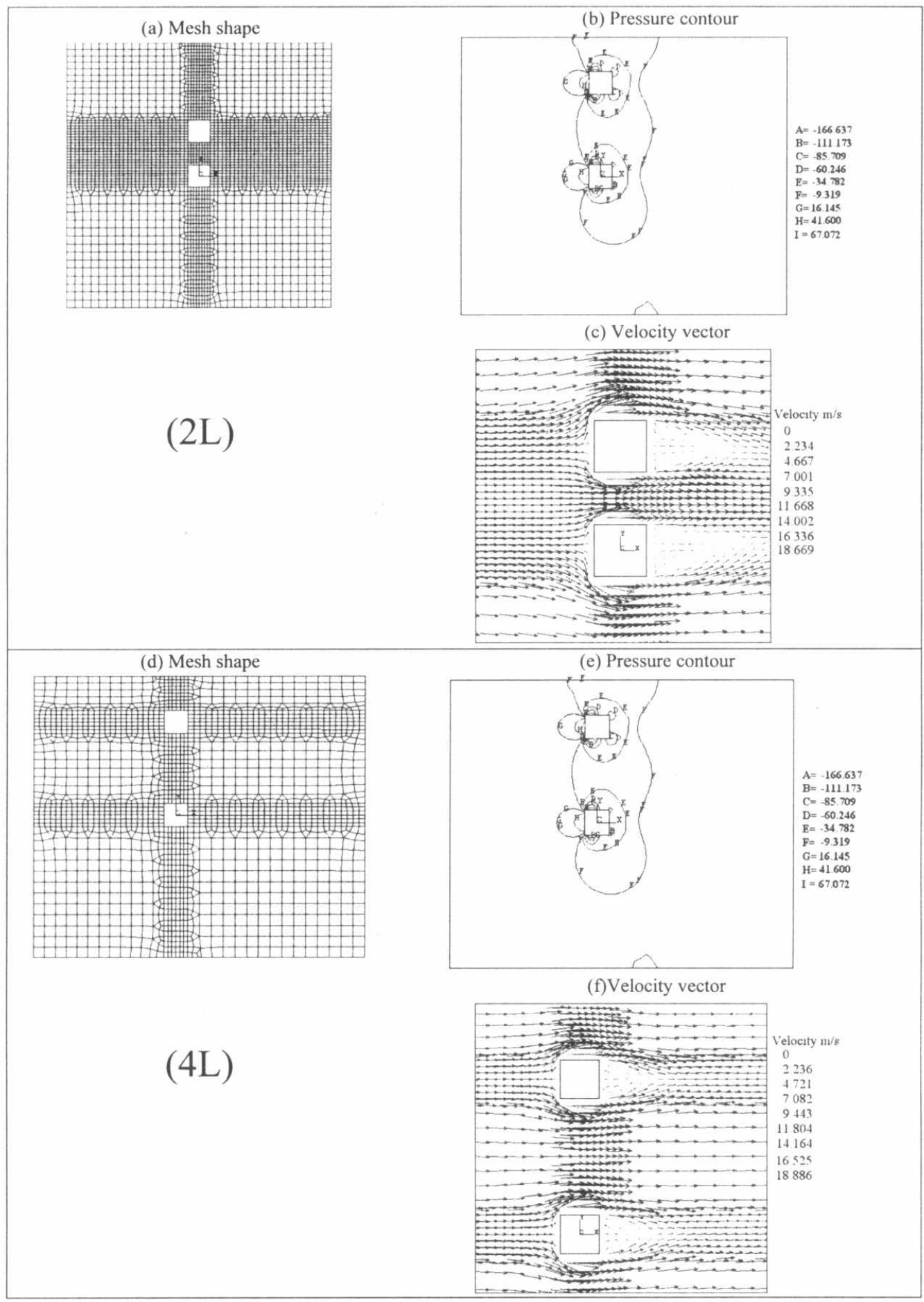

Fig. 10. Numerical results of side-by-side configuration for apart distances $2 \mathrm{~L}$ and $4 \mathrm{~L}$. 


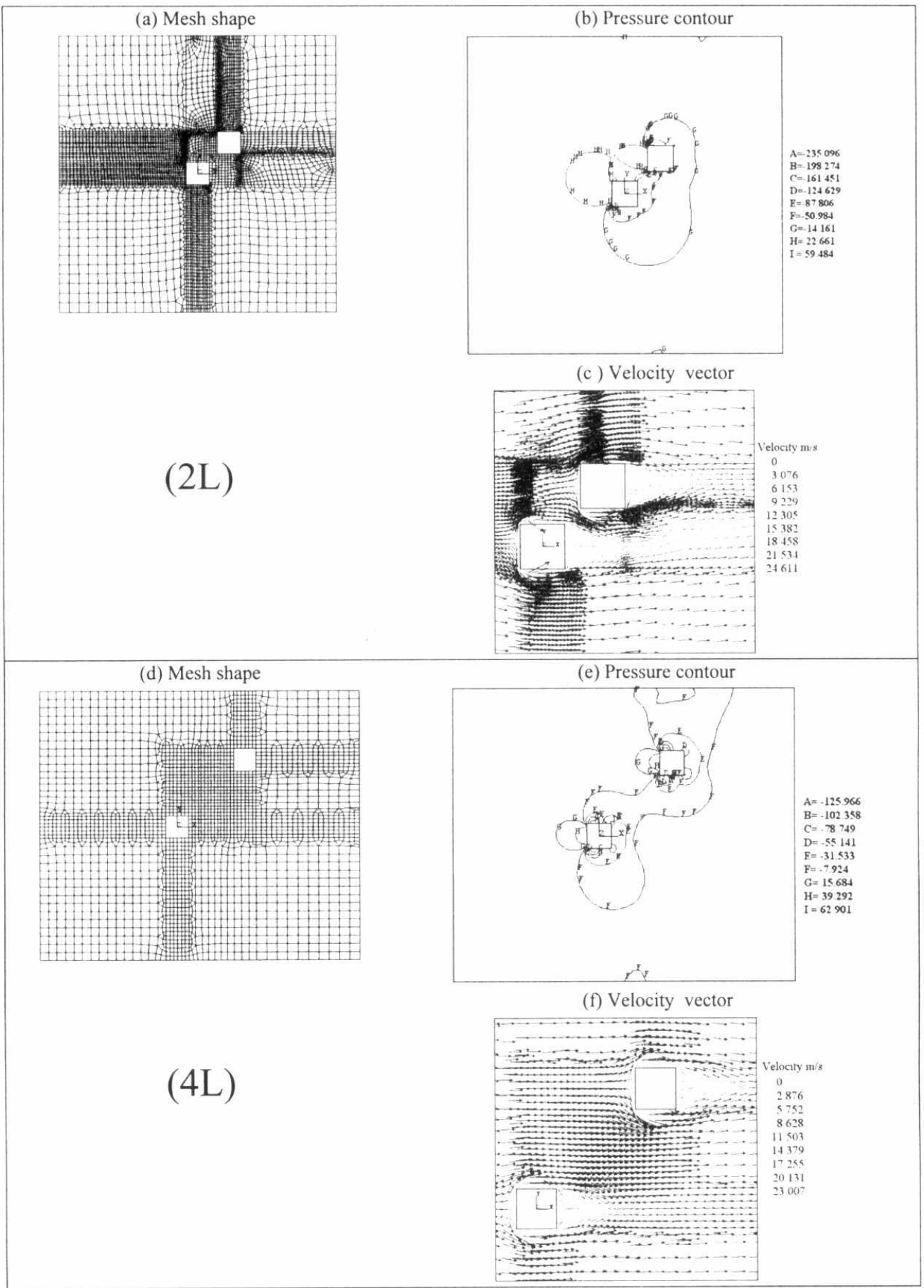

Fig. 11. Numerical results of $45^{\circ}$ staggered configuration for apart distances $2 \mathrm{~L}$ and $4 \mathrm{~L}$. 